\title{
Numerical study of bound states for point charges shielded by the response of a homogeneous two-dimensional electron gas
}

\author{
I. Nagy, ${ }^{1,2}$ M. J. Puska, ${ }^{3,2}$ and N. Zabala ${ }^{4,2,5}$ \\ ${ }^{1}$ Department of Theoretical Physics, Technical University of Budapest, H-1521 Budapest, Hungary \\ ${ }^{2}$ Donostia International Physics Center DIPC, Paseo Manuel de Lardizabal 4, 20018 San Sebastián, Spain \\ ${ }^{3}$ Laboratory of Physics, Helsinki University of Technology, P.O. Box 1100, FIN-02015 HUT, Finland \\ ${ }^{4}$ Elektrika eta Elektronika Saila, Zientzia eta Teknologia Fakultatea, UPV-EHU 644 P.K., 48080 Bilbao, Spain \\ ${ }^{5}$ Centro Mixto CSIC-UPV/EHU, Paseo Manuel de Lardizabal 4, 20018 San Sebastián, Spain
}

(Received 17 March 2006; published 25 September 2006)

\begin{abstract}
We study numerically the existence and character of bound states for positive and negative point charges shielded by the response of a two-dimensional homogeneous electron gas. The problem is related to many physical situations and has recently arisen in experiments on impurities on metal surfaces with Shockley surface states. Mathematical theorems ascertain a bound state for two-dimensional circularly symmetric potentials $V(r)$ with $\int_{0}^{\infty} d r r V(r) \leqslant 0$. We find that a shielded potential with $\int_{0}^{\infty} d r r V(r)>0$ may also sustain a bound state. Moreover, on the same footing we study the electron-electron interactions in the two-dimensional electron gas, finding a bound state with an energy minimum for a certain electron gas density.
\end{abstract}

DOI: 10.1103/PhysRevB.74.115411

PACS number(s): 73.20.Hb, 71.15.Mb

\section{INTRODUCTION AND MOTIVATION}

The properties of the two-dimensional (2D) electron gas, and in particular the phenomena induced by isolated or clustered impurities embedded in it, have attracted a large volume of experimental and theoretical research. The interest stems from the increasingly more ideal realizations of the 2D electron gas, for example, at interfaces of semiconductor heterostructures, at semiconductor surface inversion layers, at (noble) metal surfaces as Shockley surface states, or as quantum well states in metallic overlayers on insulators or other metals. At the same time the development of different photoelectron and scanning tunneling spectroscopies has enabled an increasingly more accurate characterization of these systems. ${ }^{1,2}$

Many experiments deal directly or indirectly with the existence of bound electron states in systems interacting with the 2D electron gas. The impurity-induced electron localization has been studied in the Shockley surface state systems. ${ }^{3-5}$ The quantum diffusion of hydrogen on metal surfaces reflects the coupling of the hydrogen with the metallic (possibly 2D) electron gas. ${ }^{6,7}$ Finally, a bound state between two electrons in a 2D electron gas has been proposed as an alternative pairing mechanism to the phonon coupling for high-temperature superconductivity. ${ }^{8,9}$ Remarkably, it was explicitly pointed out ${ }^{8}$ that the many-body ground state of a dilute gas of fermions is unstable to pairing if and only if a two-body bound state exists.

The background for the above-mentioned physics is laid by basic mathematical theorems. First, in two dimensions, for any everywhere attractive circularly symmetric potential $V(r)$, there exists always a bound state no matter how weak the potential is. For the case of a shallow potential valley characterized by the $\int_{0}^{\infty} d r r V(r)<0$ condition, an explicit expression for the energy eigenvalue $E_{b}$ was derived in the textbook by Landau and Lifshitz. ${ }^{10}$ It turns out that the binding energy depends exponentially on the inverse of a negative constant (given by the condition) in the investigated weak-coupling limit. The theorem of Simon ${ }^{11}$ extends the conditions for a bound state to the $\int_{0}^{\infty} d r r V(r)=0$ case, i.e., to not everywhere nonpositive or not everywhere nonnegative suitable potentials.

In this paper we present a numerical analysis for the existence of a $2 \mathrm{D}$ bound state in potentials satisfying the $\int d r r V(r)=0$ condition. The physically motivated effective potentials $V_{\text {eff }}(r)=\Lambda V(r)$ will refer to the perfectly shielded fields of embedded attractive or repulsive unit charges in a 2D electron gas, and $\Lambda$ plays the role of a convenient coupling constant for the detailed numerics. In the repulsive case the screening is constrained by the fact that the maximum of the surrounding hole density is the uniform electron gas density. In addition to the cases based on the mentioned standard condition, the overscreening and underscreening of unit charges (with $\Lambda=1$ ) will be investigated as well. The corresponding potentials could mimic the shielding dynamics in, for example, standing wave generation ${ }^{4}$ on surfaces in the presence of impurities. Slightly surprisingly, we find a bound state also for the overscreened attractive and underscreened repulsive potentials. In order to treat also the electronelectron effective interaction we use in the Schrödinger equation the reduced mass $\mu=1 / 2$. The energy of the ensuing bound electron pair has a minimum at a certain electron gas density.

The rest of the paper is organized as follows. In the next section, Sec. II, we shall deduce our physically motivated model potentials for numerical, 2D bound-state calculations. The results obtained are presented on illustrative figures, by considering relevant parameter ranges in coupling, screening tuning, and density of the electron gas. Finally, Sec. III is devoted to a short summary. Hartree atomic units $\hbar=e^{2}$ $=m_{e}=1$ will be used in the equations and discussion below.

\section{MODELS AND RESULTS}

\section{A. Shielded potentials}

We begin with a few mathematical expressions. The 2D Fourier-Hankel $(\mathrm{FH})$ transformation of a function $F(r)$ is 


$$
F(q)=2 \pi \int_{0}^{\infty} d r r J_{0}(r q) F(r)
$$

where $J_{0}(x)$ is the zeroth-order Bessel function. The inverse $\mathrm{FH}$ transform has the form

$$
F(r)=\frac{1}{2 \pi} \int_{0}^{\infty} d q q J_{0}(q r) F(q)
$$

These equations will be used below, in model-potential construction.

The field of an embedded charged particle is shielded in the $2 \mathrm{D}$ electron gas. Thus instead of the bare Coulomb form $v_{c}(q)=2 \pi / q$, one can write in momentum space

$$
V(q)= \pm v_{c}(q)[1-\Delta n(q)]
$$

for the shielded field around unit charges of different signs. Here $\Delta n(q)$ is the screening density in momentum space. With a unit norm $\Delta n(r)$ one obtains, via the above Eqs. (1) and (3), $V(q \rightarrow 0) \propto q$ limiting behavior if the real-space density decays faster than $r^{-4}$. This case corresponds to the $\int d r r V(r)=0$ condition of Simon's theorem.

Notice that the well-known ${ }^{12}$ quasiclassical ThomasFermi (TF) approximation for impurity screening in a 2D electron gas results in a monotonic potential

$$
V_{T F}(r)= \pm \int_{0}^{\infty} d q \frac{q}{q+2} J_{0}(q r)= \pm \int_{0}^{\infty} d x \frac{x e^{-2}}{\left(x^{2}+r^{2}\right)^{3 / 2}} .
$$

The negative and positive signs in Eq. (4) refer to embedded attractive and repulsive unit charges, respectively. At large distances $(r \rightarrow \infty)$ the $V_{T F}(r)$ potential falls of as $\pm 1 /\left(4 r^{3}\right)$, and one gets the $\int_{0}^{\infty} d r r V_{T F}(r)= \pm(1 / 2)$ condition. Therefore, this type of potential with negative sign belongs to the class analyzed by Landau and Lifshitz. ${ }^{10}$

We shall characterize the $\Delta n(r)$ density directly by the properly normalized hydrogenic $(\mathrm{H})$ and Gaussian $(\mathrm{G})$ forms $\left(\alpha^{2} / 2 \pi\right) \exp (-\alpha r)$ and $\left(\beta^{2} / \pi\right) \exp \left(-\beta^{2} r^{2}\right)$, respectively. The corresponding Fourier-Hankel transforms are $\alpha^{3} /\left(\alpha^{2}+q^{2}\right)^{3 / 2}$ and $\exp \left[-q^{2} /(2 \beta)^{2}\right]$, respectively. Using the former in Eq. (3) and applying Eq. (2), we get

$$
V_{H}(r)= \pm \frac{1}{r}\left\{1-2 u^{2}\left[I_{0}(u) K_{1}(u)-I_{1}(u) K_{0}(u)\right]\right\}
$$

in which $u=\alpha r / 2$ for shorthand. The Gaussian form results in

$$
V_{G}(r)= \pm \frac{1}{r}\left[1-\sqrt{2 \pi z} I_{0}(z) e^{-z}\right]
$$

where $z=\beta^{2} r^{2} / 2$. In the above equations $I_{i}(x)$ and $K_{i}(x)$ are modified Bessel functions.

We stress that $V(q=0)=0$, i.e., $\int_{0}^{\infty} d r r V(r)=0$ in these models. For long distances these shielded potentials decay as $\mp 3 /\left(2 \alpha^{2} r^{3}\right)$ and $\mp 1 /\left(4 \beta^{2} r^{3}\right)$, respectively. The attractive potentials are exhibited in Fig. 1, by fixing $\alpha=4$ (solid curve) and $\beta=2 \sqrt{2}$ (dashed curve). The $\alpha=4$ value could correspond to a strictly atomistic electron density, $1 s$-like in 2D. For a detailed comparison, two other potentials are also plot-

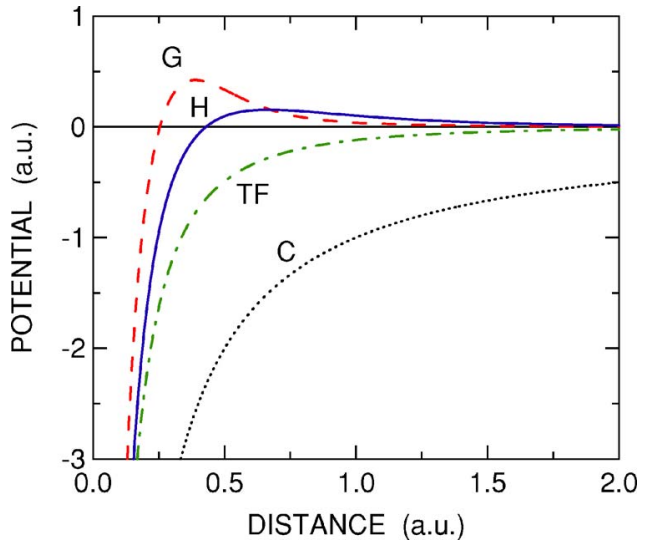

FIG. 1. (Color online) The shielded attractive potentials of Eq. (5) (solid blue curve) and Eq. (6) (dashed red curve) with $\alpha=4$ and $\beta=2 \sqrt{2}$, respectively. The dotted black curve refers to a bare Coulomb potential $-1 / r$. The dash-dotted green curve is devoted to the attractive Thomas-Fermi form of Eq. (4).

ted; that is, the bare Coulomb one $V_{c}(r)=(-1 / r)$, and the conventional Thomas-Fermi form $V_{T F}(r)$ of Eq. (4).

In order to go beyond the $\int_{0}^{\infty} d r r V(r)=0$ condition, one may multiply the shielding parts of Eqs. (5) and (6) by a variable $\lambda$ [see Eqs. (10) and (13) below]. In such a way we can investigate the overscreened $(\lambda>1)$ and underscreened $(\lambda<1)$ cases, for which $\int_{0}^{\infty} d r r V(r, \lambda) \neq 0$. Moreover, as discussed above, the effective potentials have a multiplicative coupling constant $\Lambda,\left[V_{\text {eff }}(r)=\Lambda V(r, \lambda)\right]$. In our 2D numerics $\Lambda$ and $\lambda$ will serve as convenient parameters.

\section{B. Numerical solution of the 2D Schrödinger equation}

The bound-state energy levels $\left(E_{b}\right)$ and wave functions $[\psi(r)]$ satisfy the 2D Schrödinger equation

$$
\left(-\frac{1}{2 \mu} \nabla^{2}+V_{e f f}(r)-E_{b}\right) \psi(r)=0,
$$

where $\mu$ is the reduced mass; it is unity for the impurityelectron case.

In circular symmetry the wave function separates as

$$
\psi(r)=\frac{e^{i m \phi}}{\sqrt{2 \pi}} R_{m n}(r),
$$

where $m=0, \pm 1, \pm 2, \ldots$ is the azimuthal quantum number and $n=1,2,3, \ldots$ is the radial quantum number related to the number of radial nodes $(n-1)$ of the radial wave function $R_{m n}(r)$. In this work we are interested only in the values $m$ $=0$ and $n=1$. Further, by making the substitution $U_{m n}(r)$ $=r^{1 / 2} R_{m n}(r)$ we obtain the differential equation

$$
\frac{d^{2} U_{m n}(r)}{d r^{2}}+\left(2 \mu\left[E_{b}-V_{e f f}(r)\right]-\frac{\left(m^{2}-1 / 4\right)}{r^{2}}\right) U_{m n}(r)=0 .
$$

This is the same form as the radial equation studied in spherically symmetric problems. We solve the equation on an 


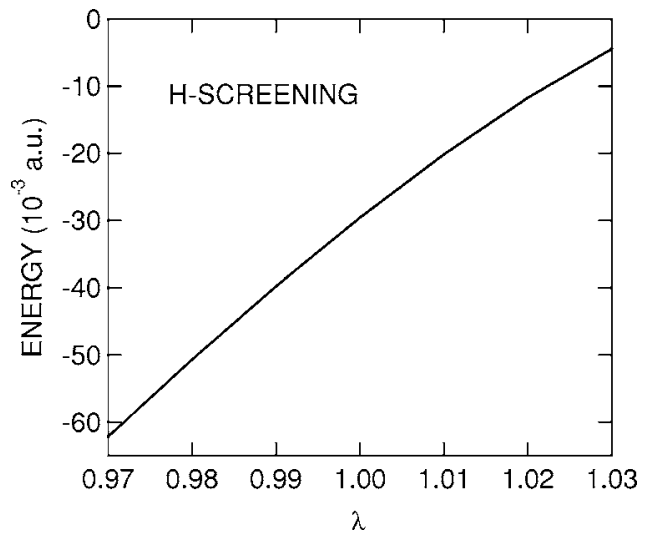

FIG. 2. Energy eigenvalue $E_{b}^{(H)}(\lambda)$ based on Eq. (7) with Eq. (10) for $\Lambda=1$ and $\lambda \in[0.97,1.03]$.

exponentially expanding radial mesh, $r(j)=r_{\min } \exp [(j$ $-1) \Delta x]$ with $j=1, \ldots, N$. With a given guess for the eigenvalue $E_{b}$ the function $U_{m n}(r)$ is integrated outward from the origin and inward from a large radius by starting with its asymptotic expansions. At a matching point close to the classical turning point the logarithmic derivatives of the outward and inward integrated solutions are required to coincide by adjusting the eigenvalue $E_{b}$. The parameters of the radial mesh, $r_{\min }, \Delta x$, and $N$, are varied until the numerical convergence of the eigenvalue is obtained.

\section{Attractive electron-ion interaction}

According to our numerical calculations, the attractive $V_{T F}(r)$ of Eq. (4) gives $E_{b}^{(T F)}=-0.2853$, while the $V_{H}(r)$ of Eq. (5) results in $E_{b}^{(H)}=-0.0296$ values for $\alpha=4$, in Hartree units. There is about an order-of-magnitude reduction in binding due to atomistic screening, beyond the quasiclassical result.

In order to study the so-called not everywhere nonpositive case (physically, the shielded positive charge) in more detail we will use, without loss of generality, $\alpha=4$ in Eq. (5) and modify it as

$$
V_{e f f}^{H}(r)=-\Lambda \frac{1}{r}\left\{1-8 \lambda r^{2}\left[I_{0}(2 r) K_{1}(2 r)-I_{1}(2 r) K_{0}(2 r)\right]\right\} .
$$

In Fig. 2 we have plotted the $E_{b}^{(H)}(\lambda)$ energies obtained with $\Lambda=1$ and $\lambda \in[0.97,1.03]$. The $\lambda$ tuning refers to small under- and overscreening. The figure clearly shows the sensitivity of binding to the details of the shielding. For $\lambda>1$ one has $\int_{0}^{\infty} d r r V_{\text {eff }}(r)>0$, with Eq. (10).

Additional information on the $\Lambda$ dependence of $E_{b}^{(H)}(\Lambda)$ for Eq. (10) and $\lambda=1$ is given in Fig. 3 with $\Lambda$ $\in[1.25,0.75]$. In this case $\int_{0}^{\infty} d r r V_{\text {eff }}(r)=0$ for any (finite) $\Lambda$, as we pointed out earlier. In harmony with Simon's theorem, ${ }^{11}\left|E_{b}(\Lambda)\right| \leqslant \exp \left(-c \Lambda^{-2}\right)$, we get for our case $\left[E_{b}^{(H)}(\Lambda)\right]$ value of $c$ of about 5.292 for the suitable constant in the investigated coupling-parameter range. The macroscopic dielectric constant of a real medium can result in re-

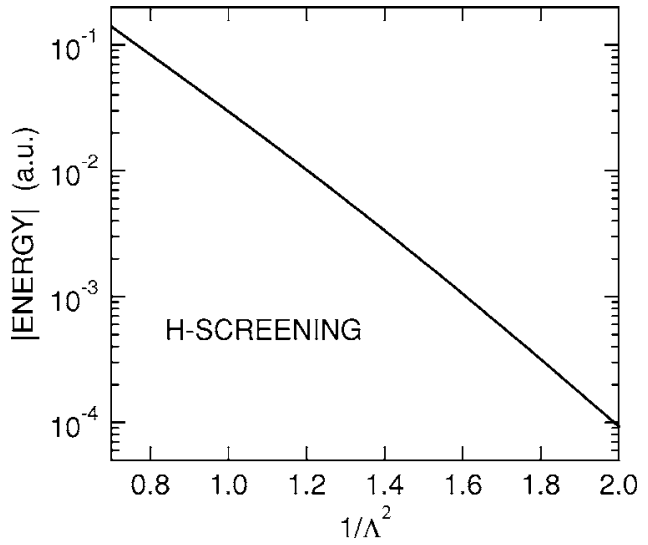

FIG. 3. Binding energy $\left|E_{b}^{(H)}(\Lambda)\right|$ based on Eq. (7) with Eq. (10) for $\lambda=1$ and $\Lambda \in[1.25,0.75]$.

duced $\Lambda<1$ values for effective interactions.

In this attractive impurity case we have shown a remarkable sensitivity of the theoretical bound-state characteristics to the shielding conditions. This is in accord with important spectroscopic information obtained ${ }^{4}$ by scanning tunneling spectroscopy of different adatoms in the standing wave patterns generated. The observed peak shift and amplitude decrease in differential tunneling conductance, as adatoms approach a step on the surface, signal the experimental sensitivity.

We note that our detailed numerical analysis is based on an effective Schrödinger equation. Further attempts are needed therefore to consider the many-body aspects of the localization problem in more detail. For example, the proper description of the width of an adatom-induced weakly bound state and its influence on scattering characteristics are important questions for future studies.

\section{Repulsive electron-electron interaction}

The so-called not everywhere nonnegative case (physically, the shielded negative charge) will be treated similarly

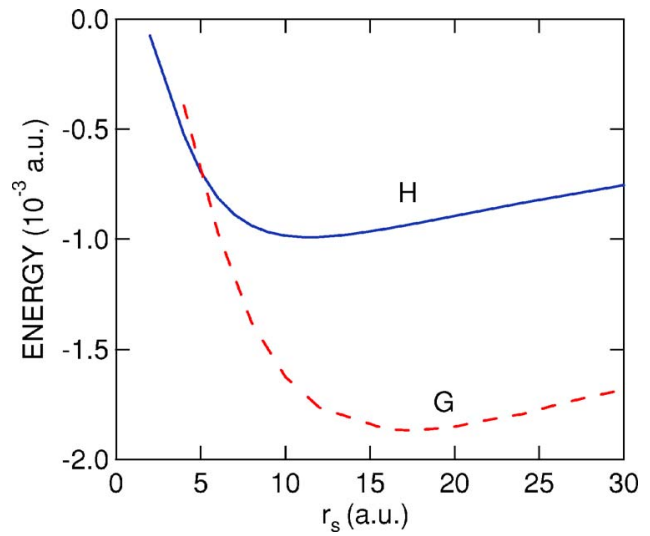

FIG. 4. (Color online) Energy eigenvalues $E_{b}\left(r_{s}\right)$ in the shielded field of a negative charge. Data are based on Eq. (7) with $\mu=1 / 2$, and $\Lambda=\lambda=1$ in the applied two effective interactions. The solid blue and dashed red curves refer to the hydrogenic [Eq. (5)] and Gaussian [Eq. (6)] models, respectively. See the text for further details. 


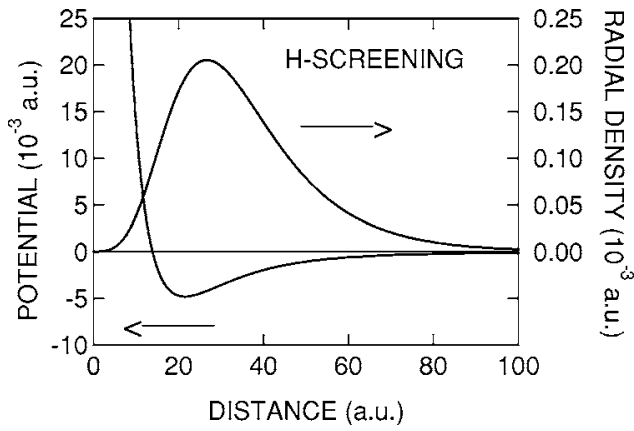

FIG. 5. The radial density $2 \pi r|\psi(r)|^{2}$ and the potential based on hydrogenic screening of a negative unit charge. These are computed at the $r_{s}=11.4$ value of the density parameter.

via Eq. (5) of positive sign, but with the constraint

$$
\lambda \alpha^{2} / 2 \pi \equiv n_{0}=1 /\left(\pi r_{s}^{2}\right)
$$

in order to model bounded, complete depletion of the 2D electron gas density at $r=0$. Here $r_{s}$ is the density parameter of the 2D gas with density $n_{0}$. The same constraint is used to reparametrize Eq. (6) (positive sign) with

$$
\lambda \beta^{2} / \pi \equiv n_{0}=1 /\left(\pi r_{s}^{2}\right)
$$

as

$$
V_{e f f}^{G}(r)=+\Lambda \frac{1}{r}\left[1-\lambda \sqrt{2 \pi z} I_{0}(z) e^{-z}\right],
$$

where, according to the above discussion, $z=\left(r / r_{s}\right)^{2} /(2 \lambda)$ now. Remember, that at $\lambda=1$ one has $\int_{0}^{\infty} d r r V_{\text {eff }}(r)=0$, independently of the value of the finite $\Lambda$.

As we use depletion-constraint-based densities to model effective electron-electron interactions, it is important to give additional physical arguments about their proper behavior. The interaction energy $(\varepsilon)$ of a repulsive point charge with a surrounding, normalized $(\lambda=1)$ shielding hole can be characterized by the classical equation

$$
\varepsilon=-\frac{1}{2} 2 \pi \int_{0}^{\infty} d r r \frac{1}{r} \Delta n(r) .
$$

This equation results in $\varepsilon_{H}=-1 /\left(\sqrt{2} r_{s}\right)$ and $\varepsilon_{G}=-\sqrt{\pi} /\left(2 r_{s}\right)$ for the exponential and Gaussian screening, respectively. These energies are between the values based on exchangeonly, $\varepsilon_{x}=-4 \sqrt{2} /\left(3 \pi r_{s}\right)$, and Wigner single-atom, ${ }^{13,14} \varepsilon_{W}$ $=-1 / r_{s}$, limiting approximations. The latter corresponds to the $\Delta n\left(r \leqslant r_{s}\right)=n_{0}$ extremum model for the hole density, and the former to the Pauli hole ${ }^{15}$ of an ideal 2D system.

The two repulsive potentials discussed above in detail are used in Eq. (7) with the reduced mass $\mu=1 / 2$, and the following numerical results are obtained. The energy eigenvalues $E_{b}\left(r_{s}\right)$ are plotted in Fig. 4, as a function of the density parameter $r_{s}$. Computed values are connected by solid (the case of exponential shielding) and dashed (the case of Gaussian model) curves. The other parameters behind these results are fixed as $\Lambda=\lambda=1$.

Remarkably, there are $r_{s}$ parameter values at which the binding energies are optimal, i.e., they have extremal values.

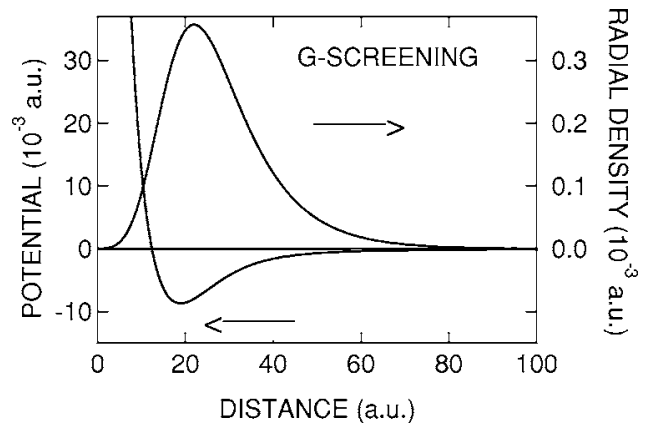

FIG. 6. The radial density $2 \pi r|\psi(r)|^{2}$ and the potential based on Gaussian screening of a negative unit charge. These are computed at the $r_{s}=17.2$ value of the density parameter.

By increasing or decreasing the density of the electron gas, the bindings become weaker. The extremal values are at about $r_{s}=11.4$ for the hydrogenlike model, and at $r_{s}=17.2$ for the Gaussian model. The binding energies are in the $10^{-3}$ range in atomic units, for the dilute system. Low carrier density and thus small Fermi energy $\varepsilon_{F}=1 / r_{s}^{2}$ are important characteristics of cuprate superconductors. ${ }^{16}$ In these materials the ratio of the critical temperature $\left(T_{c}\right)$ and the Fermi energy is in the range of $10^{-1}$. Furthermore, there is a saturation and supression of $T_{c}$ with increasing carrier density; for further detail we refer to Fig. 3 of Ref. 16.

The observed extremal character is related, physically, to our pseudononlinear construction of the effective shielding of repulsive charges; namely, the constraint via the bounded depletion hole at $r=0$ fixes a reasonable scaling in the potentials. In standard linear-response theory ${ }^{9}$ the hole density at $r=0$ can become higher than the host density $n_{0}$. In such an attempt one can get a monotonic dependence of $E_{b}\left(r_{s}\right)$ on $r_{s}$ in the resulting shielded fields.

Further information is given in Figs. 5 and 6, which show the 2D radial densities $2 \pi r|\psi(r)|^{2}$ computed with bound-state wave functions at the extremal Wigner-Seitz parameters. The corresponding potentials are also plotted. As expected, the square-integrable wave functions are localized at about the potential minima. In the investigated equal-mass case this

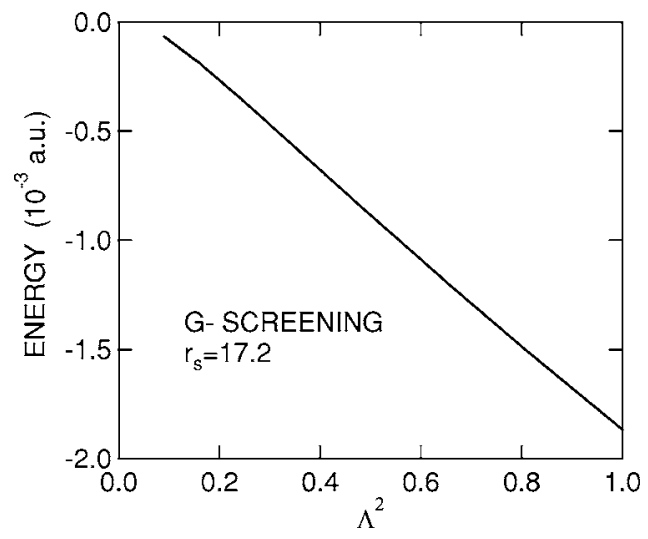

FIG. 7. The coupling-constant $(\Lambda)$ dependence of the energy eigenvalue for the repulsive case. The results are based on the Gaussian model of Eq. (13) with $\mu=1 / 2$ in Eq. (7). The WignerSeitz parameter is $r_{s}=17.2$ and $\lambda=1$. 


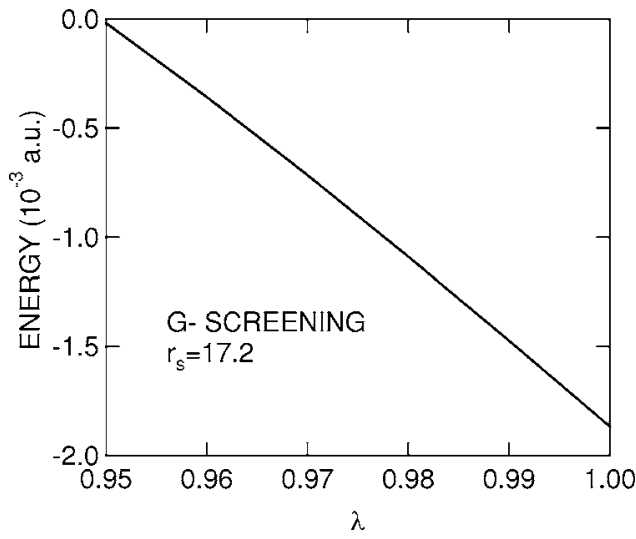

FIG. 8. The shielding-constant $(\lambda)$ dependence of the energy eigenvalue for the repulsive case. The results are based on the Gaussian model of Eq. (13) with $\mu=1 / 2$ in Eq. (7). The WignerSeitz parameter is $r_{s}=17.2$ and $\Lambda=1$.

extension can represent a certain coherence length; somewhat surprisingly it is only twice the extremal density parameter.

As we observed in Fig. 3 for the attractive case, the binding energy depends on the coupling $\Lambda$. An illustration of this fact for the repulsive case is given in Fig. 7. The Gaussian model of Eq. (13) is used with $\lambda=1, \mu=1 / 2$, and $r_{s}=17.2$. Results for $\Lambda<1$ are plotted. We can approximate our data by a quadratic expression, i.e., $\left|E_{b}^{(G)}(\Lambda)\right| \sim \Lambda^{2}$. Rescaling of $\Lambda=1$ by a macroscopic dielectric constant could result in a notable reduction of the above-mentioned binding energies (see Fig. 4 also).

We finish our representation by discussing the question of undershielding the repulsive charge. Figure 8 is devoted to this problem. We have used Eq. (13) with $\Lambda=1$ and $\lambda<1$ in the Schrödinger equation (7) with $\mu=1 / 2$, at $r_{s}=17.2$. One can observe (see Fig. 2 for the attractive case) essential reductions of energies for the $\int_{0}^{\infty} d r r V(r)>0$ unconventional condition.

The last results of Figs. 7 and 8, together with Figs. 4-6, signal a nontrivial sensitivity of the magnitude of the binding energy on the concrete physical situation. Fortunately, there is a physical limitation. It should be clear from the discussion of Eq. (14), and as our illustrative figures indeed show, that a more localized real-space character of the bounded (and normalized) depletion hole around a repulsive unit charge results in an effective potential with a repulsive part of shorter range. Clearly, the limitation is given by the Wigner model, in which one has the $\Delta n\left(r \leqslant r_{s}\right)=n_{0}$ extremum for the hole.

\section{SUMMARY}

In this work we have investigated the problem of bound states in two-dimensional shielded potentials. Effective potentials, based on direct approximation for the screening charge densities around attractive and repulsive unit charges, are employed. In particular, the effective electron-electron interaction is modeled via a properly constrained depletion hole. In the detailed numerical analysis performed, we found that in both the basically attractive and basically repulsive potentials bound states appear under the $\int_{0}^{\infty} d r r V(r)=0$ standard, and may appear under the $\int_{0}^{\infty} d r r V(r)>0$ unconventional conditions. In the repulsive case the extremal character of the binding energy, as a function of the density of the host 2D electron gas, is established with our physical models for effective electron-electron interactions.

\section{ACKNOWLEDGMENTS}

I.N. and M.J.P. are thankful for the warm hospitality at DIPC, where this study was completed. They gratefully acknowledge useful discussions with P. M. Echenique. The work of I.N. has been supported partly by the Hungarian OTKA (Grants No. T046868 and No. T049571), that of M.J.P. by the Academy of Finland, and that of N.Z. by the University of Basque Country (Grant No. 9/UPV00206.21513639/2001) and the Basque Unibertsitate eta Ikerketa Saila, the MCyT (Grant No. FIS2004-06490-C03-00), and the EU Network of Excellence NANOQUANTA (Grant No. NMP4CT-2004-500198).
${ }^{1}$ S. Hüfner, Photoelectron Spectroscopy-Principles and Applications, Springer Series in Solid-State Science Vol. 82 (Springer, Berlin, 1995).

${ }^{2}$ P. M. Echenique, R. Berndt, E. V. Chulkov, Th. Fauster, A. Goldman, and U. Höfer, Surf. Sci. Rep. 52, 219 (2004).

${ }^{3}$ F. E. Olsson, M. Persson, A. G. Borisov, J. P. Gauyacq, J. Lagoute, and S. Fölsch, Phys. Rev. Lett. 93, 206803 (2004).

${ }^{4}$ L. Limot, E. Pehlke, J. Kröger, and R. Berndt, Phys. Rev. Lett. 94, 036805 (2005).

${ }^{5}$ C. Liu, I. Matsuda, R. Hobara, and S. Hasegawa, Phys. Rev. Lett. 96, 036803 (2006).

${ }^{6}$ L. J. Lauhon and W. Ho, Phys. Rev. Lett. 85, 4566 (2000).
${ }^{7}$ J. Kondo, Physica B \& C 125B, 279 (1984).

${ }^{8}$ M. Randeria, J. M. Duan, and L. Y. Shieh, Phys. Rev. Lett. 62, 981 (1989).

${ }^{9}$ A. Ghazali and A. Gold, Phys. Rev. B 52, 16634 (1995).

${ }^{10}$ L. D. Landau and E. M. Lifshitz, Quantum Mechanics (AddisonWesley, Reading, MA, 1958).

${ }^{11}$ B. Simon, Ann. Phys. (N.Y.) 97, 279 (1976).

${ }^{12}$ F. Stern and W. E. Howard, Phys. Rev. 163, 816 (1967).

${ }^{13}$ E. Wigner, Phys. Rev. 46, 1002 (1934).

${ }^{14}$ I. Nagy, Phys. Rev. B 60, 4404 (1999).

${ }^{15}$ M. Seidl, J. P. Perdew, and M. Levy, Phys. Rev. A 59, 51 (1999).

${ }^{16}$ Y. J. Uemura et al., Phys. Rev. Lett. 66, 2665 (1991). 